\title{
The Second Meal Effect and Its Influence on Glycemia
}

\section{Justin A Fletcher ${ }^{1,4}$, James W Perfield II ${ }^{1,2}$, John P Thyfault ${ }^{1,3,4}$ and R Scott Rector ${ }^{1,3,4 *}$}

${ }^{1}$ Departments of Nutrition and Exercise Physiology, University of Missouri, USA

${ }^{2}$ Food Science of Gastroenterology and Hepatology, University of Missouri, USA

3Internal Medicine-Division of Gastroenterology and Hepatology, University of Missouri, USA

${ }^{4}$ Harry S Truman Memorial Veterans Medical Center, Columbia, Missouri 65201, USA

\begin{abstract}
The study of factors that influence blood glucose homeostasis is becoming increasingly important as prevalence rates for insulin resistance and type 2 diabetes have increased. Diets with a low glycemic index (lowGI) have been shown to reduce the risk for diseases by limiting the increase of glucose in the blood. LowGl intake in one meal also has been shown to limit the postprandial glycemic response (PPGR) to a subsequent meal; a concept termed the "second meal effect". Although there have been many theories for the mechanisms responsible for the second meal effect, the exact cause has yet to be elucidated. It is important for both research investigators as well as patients to consider food consumption prior to testing PPGR, as there is evidence that the GI of one meal not only affects the PPGR of that meal, but will also influence the PPGR of the following meal(s). Here we will review recent evidence regarding factors believed to contribute to the second meal effect and the magnitude of their impact.
\end{abstract}

Keywords: Glycemic index; Glycemic load; Postprandial glycemic response; Glycemia; Second meal effect; Glycogen

\section{Introduction}

It is vital for individuals with diabetes or other metabolic conditions to control blood glucose in order to prevent the development of cardiovascular disease and other pathological conditions. In fact, the first goal listed in the American Diabetes Association's 2008 position statement is for those with diabetes to properly maintain blood glucose within a narrow range [1]. Careful attention to diet is essential for maintaining controlled blood glucose levels. Research has shown that eating meals containing high glycemic loads and low amounts of fiber can increase an individual's risk for developing type 2 diabetes [2,3].

The increase in blood glucose levels after a meal is termed postprandial glycemic response (PPGR). PPGR is a measure that can be used to gauge the competency of the metabolic system. Essentially, postprandial blood glucose concentration is controlled by two components, glucose appearance and glucose clearance [1,4-6]. Glucose appearance and the magnitude of the glycemic response are affected by the speed at which a carbohydrate is digested and enters the blood stream. A healthy human body is able to minimize PPGR and maintain euglycemia. An important process in removing glucose from the blood after a meal is the activation of the insulin signaling pathway, and subsequent glucose transport into various peripheral tissues including skeletal muscle and adipose tissue. The most important tissue for glucose removal is skeletal muscle, which has been shown to be responsible for $85 \%$ of the body's insulin-stimulated glucose clearance [7].

There have been attempts to create a method of measuring/ predicting a food's ability to impact glycemic response [8]. Jenkins et al. [9] developed a concept known as the glycemic index (GI) to classify a food based on a PPGR. Wolever and Bolognesi C [10] defines GI "as the incremental area under the glucose response curve for a $50-\mathrm{g}$ carbohydrate portion of a food expressed as a percentage of that after $50 \mathrm{~g}$ carbohydrate of white bread is taken by the same subject". Although there are multiple ways to express area under the curve [11], the most common method at it relates to GI is the incremental area under the curve ignoring the area under the baseline [11]. Said another way, GI is determined by measuring the increase in blood glucose concentration above fasting levels over a two-hour period after consuming a food that usually contains 50 grams of carbohydrate [8]. This value is then divided by the glycemic response to a reference food such as 50 grams of glucose or white bread and multiplied by $100[1,4]$. An additional measure is termed glycemic load (GL), which takes into account both the GI of the food and the amount of carbohydrate in the food. GL is calculated by first dividing the GI by 100 then multiplying this value by the carbohydrate content (grams) followed by multiplying by, the total weight of the food. In summary, the higher the GI value of a food, the more it is expected to increase blood glucose levels [8].

It is currently estimated that there are 26.8 million individuals in the United States with type 2 diabetes mellitus [12]. With diabetes becoming more prevalent, there is a need for studies on glycemic response to food items and GI. These systems of classifying foods based on their impact on PPGR can be very helpful to diabetics who need to carefully monitor their blood glucose concentrations [13].

One factor known to affect glycemia has been termed "the second meal effect". The second meal effect is a phenomenon where the GI of one meal can influence the glycemic response to a subsequent meal $[14,15]$. This concept is illustrated in Figure 1. For instance, a low glycemic index (lowGI) breakfast has been shown to lower the PPGR to lunch [14]. This effect also has been shown to last overnight with the GI of an evening meal affecting the PPGR to a breakfast meal the following morning [16]. If not recognized and controlled for by the investigator, the second meal effect may influence the results of studies determining the effects of particular food items on glycemia. The purpose of this review is to investigate the influence of the second meal effect in studies researching PPGR and examine the factors that contribute to the second meal effect.

*Corresponding author: R. Scott Rector, PhD, Assistant Professor and Research Health Scientist, Harry S Truman Memorial VA Hospital, Departments of Internal Medicine - Division of Gastroenterology and Hepatology and Nutrition and Exercise Physiology, University of Missouri-Columbia, Columbia, MO 65212, USA, Tel: 573884-0979; Fax: 573-884-4595; E-mail: rectors@health.missouri.edu

Received February 01, 2012; Accepted March 01, 2012; Published March 03, 2012

Citation: Fletcher JA, Perfield II JW, Thyfault JP, Rector RS (2012) The Second Meal Effect and Its Influence on Glycemia. J Nutr Disorders Ther 2:108. doi:10.4172/2161-0509.1000108

Copyright: (C) 2012 Fletcher JA, et al. This is an open-access article distributed under the terms of the Creative Commons Attribution License, which permits unrestricted use, distribution, and reproduction in any medium, provided the original author and source are credited. 
First Meal

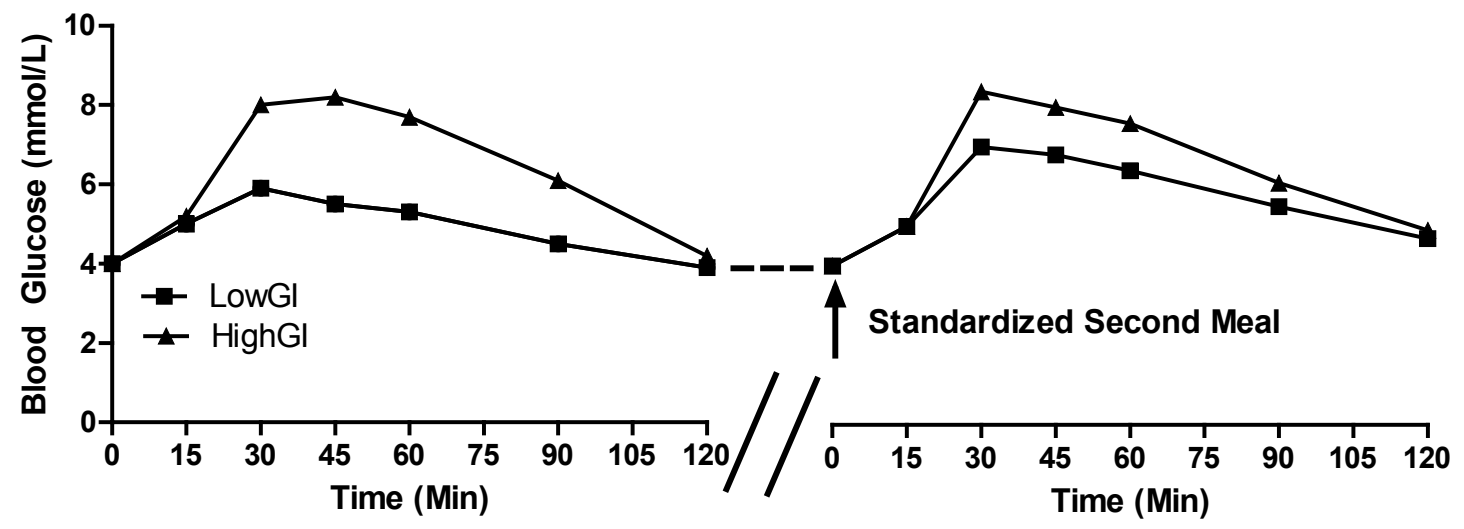

Figure 1: Example of blood glucose curves to a first and second meal in response to meals of high and low glycemic indices. A meal with a low glycemic index (lowGl) has a lower postprandial glycemic response during the first meal compared with a high glycemic index (highGl) meal and is capable of lowering the glycemic response to a standardized second meal.

\section{Glycemic index}

The observation of a second meal effect was reportedly discovered in the early $20^{\text {th }}$ century $[17,18]$. Research on the second meal effect has recently become more popular due to the fact that poor glycemic control is linked to multiple health complications [19]. In 1988, Wolever et al. [15] studied the impact of meals with differing GIs on the second meal effect. The subjects consumed 2 different evening meals on separate occasions, with one of high (GI:105) and the other of low (GI:40.6) glycemic index. Glycemic response was then measured the next day following breakfast, to determine if the GI of an evening meal affected the glycemic response of the subsequent morning meal. It was found that evening meals with a lowGI significantly lowered the mean PPGR to a breakfast meal the next morning compared to a meal with a highGI (6.36 vs. 6.91 respectively; $\mathrm{p}<0.05)$.

\section{Fiber}

Recent research suggests that fiber may be a significant contributing factor that impacts PPGR, independent of the GI [20]. Fiber can help reduce the PPGR to a meal by slowing down the digestion rate or by reducing the amount of glucose that is absorbed in the small intestine [21]. Fiber and indigestible carbohydrates (resistant starch and dietary fiber) also have been shown to reduce the PPGR to a subsequent meal. This was examined by Granfeldt et al. [20] who compared three different evening meals with varying carbohydrate content (barley kernels, spaghetti, and white bread) to examine the effects of indigestible carbohydrates on GI and the influence each meal had on the PPGR to a breakfast meal the following morning. A spaghetti meal made of durum wheat was found to have a GI of 54, while the barley kernel meal had a GI of 53. The third meal, the reference white bread, had a GI of 100. The authors found that the barley meal, which contained high levels of indigestible carbohydrates, significantly reduced $(-23 \%)$ the PPGR to the standardized breakfast meal compared to white bread or spaghetti, and concluded the reduction was due to the higher amounts of indigestible carbohydrates [20].

Indigestible carbohydrates have become a common explanation among researchers for the second meal effect. One mechanism by which indigestible carbohydrates is believed to be able to mitigate the glycemic response to a subsequent meal (second meal effect) is through short chain fatty acids (SCFA) created by colonic fermentation
$[14,22,23]$. It has been shown that a spaghetti meal with a GI of 58 prior to $19.8 \mathrm{~g}$ of barley dietary fiber content being added resulted in a significantly lower glucose AUC during a second meal compared to the barley porridge meal (GI:97) [24]. In addition, the spaghetti with twice the barley dietary fiber content meal also led to significantly higher colonic fermentation as assessed by breath hydrogen, as well as butyrate (SCFA) levels compared to the barley porridge meal. The authors theorized that the SCFAs were responsible for the reduction in the PPGR to the standardized breakfast meal. Evidence supporting this theory includes a negative correlation between plasma butyrate and propionate with glycemic measurements. However, there also was a positive correlation between fasting free fatty acid (FFA) levels and PPGR to the breakfast meal. The authors suggest that the FFAs influenced insulin sensitivity and therefore the glycemic response to the breakfast meal. While this study did not provide evidence of a link between SCFAs and FFAs, other studies have suggested that SCFAs may suppress FFA concentration levels [22,25].

Brighenti et al. [22] also investigated the effects of indigestible carbohydrates on PPGR in a study that examined breakfast meals of lowGI, highGI, and highGI with lactulose sponge cakes. Lactulose was added to the third test meal due to the fact that it is indigestible yet highly fermentable. The authors found that PPGR in response to the breakfast meal was significantly lower for the lowGI meal compared to the other two treatments. The highGI with lactulose breakfast resulted in significantly lower PPGR at the 3 and 4 hour poststandardized lunch time periods compared to the highGI breakfast. The authors hypothesized that SCFAs may be responsible for delayed gastric emptying and that a delay in gastric emptying could cause the second meal effect. However, this effect was only significant in the lactulose group, while both the highGI with lactulose and lowGI breakfast resulted in colonic fermentation. In addition, the glycemic measurements were not correlated with the rate of gastric emptying. Therefore, the delayed gastric emptying is believed to only play a secondary role in the influence on the second meal effect. Instead, it is believed that the SCFAs formed through colonic fermentation suppressed FFAs, therefore aiding in glucose disposal and the lowering of the PPGR to the lunch meal [22].

Liljeberg et al. [26] also observed a second meal effect on glycemic control, but came to a different conclusion as to what was responsible 
for the effect. The authors found that 2 of the 4 lowGI meals lowered blood glucose levels following a subsequent meal. Of those two meals one had a high indigestible carbohydrate content the other had a low content. It also was found that the spaghetti meal, which had the lowest PPGR to lunch, also had the fewest indigestible carbohydrates. In addition, two highGI meals that had higher levels of indigestible carbohydrates failed to cause a second meal effect. This led the authors to believe that fermentable carbohydrates are not responsible for the second meal effect. Instead, they suggest that the lowGI of the meal is responsible for the second meal effect, although it is evident that not all meals of lowGIs are equal in inducing the second meal effect.

In summary, while not all studies are in complete agreement, it appears that indigestible carbohydrates in lowGI foods are at least partially responsible for the second meal effect. Although the exact mechanism behind the second meal effect has yet to be elucidated, delayed gastric emptying caused by short chain fatty acids, and improved insulin sensitivity are two other possibilities that have been proposed.

\section{Lactic acid}

Lactic acid is produced during fermentation and has been shown to reduce PPGR to a single meal [27,28]. Najjar et al. [29] assessed the presence of organic acids (lactic acid) by measuring the $\mathrm{pH}$ of the bread and found that the sourdough bread had a lower $\mathrm{pH}$ than the white bread, indicating a greater amount of lactic acid. The authors also reported that the sourdough bread eaten as a breakfast meal resulted in a significantly lower PPGR to a lunch meal compared to the whole wheat and whole wheat barley breakfast meals. The sourdough bread breakfast also resulted in a significantly lower incretin (GLP-1 and GIP) response to the lunch meal compared to the other test breads. While insulin sensitivity has been shown to be increased by incretins (GLP-1) [30], this study did not find any significant difference in insulin response between the sourdough bread and the other test breads. Nor did they find any differences in insulin sensitivity index among the different test breads. Therefore, it is unlikely that changes in insulin sensitivity were responsible for the lower PPGR to the subsequent meal after a sourdough bread breakfast. Previous studies also have shown that organic acids may slow the rate of gastric emptying [28,31,32]. However, Najjar et al. [29] assessed gastric emptying through paracetamol absorption studies and found no significant difference between the sourdough bread and the other tests breads indicating a reduction in the rate of gastric emptying was not responsible for the reduced PPGR in these studies. In the end, the authors found a link between the sourdough bread and organic acids, and the second meal effect, but were unable to elucidate the exact mechanism behind the lower PPGR to a subsequent meal.

In another study examining the influence of lactic acid on the PPGR to a second meal, Ostman et al. [33] compared the impact of barley bread with and without lactic acid on the PPGR to a subsequent lunch meal. Consuming barley bread containing lactic acid at breakfast significantly reduced the glycemic response after breakfast and lunch when compared to the control breakfast. The authors suggested that prolonged starch digestion may have suppressed the concentration of FFAs in the plasma, which may aid insulin sensitivity as suggested by Wolever et al. [34]. Another possible explanation is that the lactic acid aided in the formation of SCFAs through colonic fermentation, which may impact gastric motility. However, due to the fact that there were only 4 hours between breakfast and lunch it is unlikely that this was enough time to ferment SCFAs [33]. Therefore, while there is evidence that consuming a meal containing lactic acid can aid in lowering the
PPGR to a subsequent meal the exact mechanism responsible for this effect is unclear.

\section{Glycogen and insulin}

Another possible explanation of the second meal effect is that it is caused by the suppression of FFAs and glucose being stored as glycogen. Jovanovic et al. [19] studied the postprandial glycemic and insulemic responses to a standard lunch meal on two separate occasions; with and without breakfast being consumed that same day. Glycogen was measured using ${ }^{13} \mathrm{C}$ magnetic resonance spectroscopy before lunch. Then 3 grams of $\left[\mathrm{U}-{ }^{13} \mathrm{C}\right]$ glucose was given at lunch and glycogen was measured again after lunch 2 and 5 hours later. It was found that eating breakfast suppressed FFAs and reduced the glycemic response $(73 \%)$ to a standard lunch compared to not eating breakfast. It also was found that fasting glycogen levels were similar on days with and without breakfast. However, on days when breakfast was consumed, there was approximately $50 \%$ more glucose contributing to the formation of glycogen within 2 hours of eating lunch compared to days when no breakfast was eaten. Furthermore, this value was doubled within 5 hours of lunch on breakfast days. There also was an inverse correlation between FFA levels prior to lunch and glycogen signaling after lunch. The authors hypothesized that insulin release following a breakfast meal suppressed FFA concentrations, as well as increased insulin action, skeletal muscle glucose uptake, and glycogen storage.

\section{Other blood markers}

There is evidence that the second meal effect involves cytokines and hormones other than insulin. Nilsson et al. [23] reported that consuming an evening meal of ordinary barley kernel bread (GI:52) resulted in significantly lower concentrations of glucose, insulin, IL-6, and FFAs, as well as higher levels of GLP-1 and adiponectin compared to the white-wheat bread meal (GI:100). The authors speculated that a reduction in FFAs was responsible for the improvements seen in glucose tolerance the next morning, as FFAs are known to impair insulin sensitivity [35]. These improvements in glucose tolerance were thought to be in part through the insulin sensitizing effects of increased adiponectin and its ability to lower FFAs in circulation [36] and the reductions in IL-6, a known activator of adipose tissue lipolysis [37]. In addition, increases in GLP-1, which has been shown to reduce gastric emptying and increase insulin release [38], may also be a contributing factor in the second meal effect. Furthermore, breath hydrogen excretion revealed a potential role for colonic fermentation of indigestible carbohydrates and the accumulation of SCFAs. These all appear to be possible mechanisms that may have contributed to the lower FFA values and second meal effect seen in the ordinary barley kernel bread compared to the white wheat bread meals.

\section{Second meal effect and type 2 diabetes}

Although many of the studies presented in this review have utilized healthy subjects, the second meal effect has also been observed in subjects with insulin resistance and type 2 diabetes. Clark et al [39] examined how high (GI:64) and lowGI (GI:56) breakfast meals influence the glycemic response to a standardized lunch in individuals diagnosed with type 2 diabetes. The lowGI meal, which contained psyllium soluble fiber, resulted in a significantly lower PPGR to the breakfast meal compared to the highGI breakfast meal but these differences did not persist after lunch. The same findings were observed for insulin and FFAs. While the lowGI breakfast meal containing psyllium fiber offered acute benefits in glucose, insulin, and FFA AUC values, it did not induce a second meal effect. These findings contradict those of Axelsen et al. [40] who found that carbohydrates in the form 
of uncooked starch (slow digesting starch) consumed as an evening meal improved glycemic response in individuals with type 2 diabetes to a breakfast meal compared to white bread. Jovanovic et al. [41] also found that in individuals with type 2 diabetes, eating breakfast can lower PPGR to a lunch meal compared to not eating breakfast. They also reported that while eating breakfast did not influence insulin levels in individuals with type 2 diabetes, it did suppress FFA levels, which correlated with glucose AUC values. These findings suggest that FFAs may play a role in the second meal effect in this population.

\section{Duration of the second meal effect}

Knowing the duration of the second meal effect can be important, not only for individuals with type 2 diabetes, but also for healthy individuals trying to control their glucose excursions. Jenkins et al. [42] determined the Staub-Traugott effect, now called the second meal effect, lasted from breakfast to lunch (a 4 hour period) by showing that slow digesting carbohydrate breakfast meals improved glucose tolerance during a subsequent lunch. Others have shown that lowGI foods consumed in the evening can improve the glucose tolerance to a breakfast meal the following morning [15]. However, Nilsson et al. [14] found that the effect did not last from breakfast to dinner (9.5 hours). Therefore, additional information is still required to understand the dietary and population specific factors that may influence the duration of the second meal effect.

\section{Summary and Conclusions}

While the second meal effect is beginning to receive attention, it is likely that many health care providers and researchers are still unaware of this phenomenon. The second meal effect appears to be related to several mechanisms. Eating foods with lowGIs and indigestible carbohydrates have been shown to reduce postprandial glycemic responses, and can be a very useful tool for diabetes management. In addition, researchers conducting glycemic testing to meals or glucose challenges should understand the impact the second meal effect can have on their testing. Failure to carefully monitoring the GI and indigestible carbohydrate content of a subject's evening meals prior to morning testing may have profound effects on the results. As important and useful as the knowledge of the second meal effect is, it is important to continue research in this area and elucidate the exact mechanisms responsible.

\section{References}

1. American Diabetes Association, Bantle JP, Wylie-Rosett J, Albright AL, Apovian CM, et al. (2008) Nutrition recommendations and interventions for diabetes: a position statement of the American Diabetes Association. Diabetes Care 31 Suppl 1: S61-S78.

2. Salmerón J, Ascherio A, Rimm EB, Colditz GA, Spiegelman D, et al. (1997) Dietary fiber, glycemic load, and risk of NIDDM in men. Diabetes Care 20: $545-550$.

3. Salmeron J, Manson JE, Stampfer MJ, Colditz GA, Wing AL, et al. (1997) Dietary fiber, glycemic load, and risk of non-insulin-dependent diabetes mellitus in women. JAMA 277: 472-477.

4. Hlebowicz J (2009) Postprandial blood glucose response in relation to gastric emptying and satiety in healthy subjects. Appetite 53: 249-252.

5. Schenk S, Davidson CJ, Zderic TW, Byerley LO, Coyle EF (2003) Differen glycemic indexes of breakfast cereals are not due to glucose entry into blood but to glucose removal by tissue. Am J Clin Nutr 78: 742-748.

6. Sheard NF, Clark NG, Brand-Miller JC, Franz MJ, Pi-Sunyer FX, et al. (2004) Dietary carbohydrate (amount and type) in the prevention and management of diabetes: a statement by the american diabetes association. Diabetes Care 27: 2266-2271

7. McCoy M, Proietto J, Hargreves M (1994) Effect of detraining on GLUT-4 protein in human skeletal muscle. J Appl Physiol 77: 1532-1536.
8. Monro JA Shaw M (2008) Glycemic impact, glycemic glucose equivalents, glycemic index, and glycemic load: definitions, distinctions, and implications. Am J Clin Nutr 87: 237S- 243S

9. Jenkins DJ, Wolever TM, Taylor RH, Barker H, Fielden H, et al. (1981) Glycemic index of foods: a physiological basis for carbohydrate exchange. Am J Clin Nutr 34: 362-366.

10. Wolever TM, Bolognesi C (1996) Source and amount of carbohydrate affect postprandial glucose and insulin in normal subjects. J Nutr 126: 2798-2806.

11. Brouns F, Bjorck I, Frayn KN, Gibbs AL, Lang V, et al. (2005) Glycaemic index methodology. Nutr Res Rev 18:145-171.

12. Shaw JE, Sicree RA, Zimmet PZ (2010) Global estimates of the prevalence of diabetes for 2010 and 2030. Diabetes Res Clin Pract 87: 4-14.

13. Barclay AW, Petocz P, McMillan-Price J, Flood VM, Prvan T, et al. (2008) Glycemic index, glycemic load, and chronic disease risk--a meta-analysis of observational studies. Am J Clin Nutr 87: 627-637.

14. Nilsson AC, Ostman EM, Granfeldt Y, Bjorck IM (2008) Effect of cereal test breakfasts differing in glycemic index and content of indigestible carbohydrates on daylong glucose tolerance in healthy subjects. Am J Clin Nutr 87: 645-654.

15. Wolever TM, Jenkins DJ, Ocana AM, Rao VA, Collier GR (1988) Second-mea effect: low-glycemic-index foods eaten at dinner improve subsequent breakfast glycemic response. Am J Clin Nutr 48: 1041-1047.

16. Stevenson E, Williams C, Nute M, Humphrey L, Witard O (2008) Influence of the glycaemic index of an evening meal on substrate oxidation following breakfast and during exercise the next day in healthy women. Eur J Clin Nutr 62: $608-616$

17. Staub H (1921) Examination of sugar metabolisms in humans. Z Klin Med 91 44-48.

18. Traugott K (1922) In reference to the reactions of blood sugar levels in repeated and varied types of enteral sugar increases and their significance in liver function. Klin Wochenschr 1: 892-894.

19. Jovanovic A, Leverton E, Solanky B, Ravikumar B, Snaar JE, et al. (2009) The second-meal phenomenon is associated with enhanced muscle glycogen storage in humans. Clin Sci (Lond) 117: 119-127.

20. Granfeldt Y, Wu X, Bjorck I (2006) Determination of glycaemic index; some methodological aspects related to the analysis of carbohydrate load and characteristics of the previous evening meal. Eur J Clin Nutr 60: 104-112.

21. Jenkins DJ, Wolever TM, Nineham R, Sarson DL, Bloom SR, et al. (1980) Improved glucose tolerance four hours after taking guar with glucose. Diabetologia 19: 21-24

22. Brighenti F, Benini L, Del Rio D, Casiraghi C, Pellegrini N, et al. (2006) Colonic fermentation of indigestible carbohydrates contributes to the second-meal effect. Am J Clin Nutr 83: 817-822.

23. Nilsson AC, Ostman EM, Holst JJ, Bjorck IM (2008) Including indigestible carbohydrates in the evening meal of healthy subjects improves glucose tolerance, lowers inflammatory markers, and increases satiety after a subsequent standardized breakfast. J Nutr 138: 732-739.

24. Nilsson A, Ostman E, Preston T, Bjorck I (2008) Effects of GI vs conten of cereal fibre of the evening meal on glucose tolerance at a subsequent standardized breakfast. Eur J Clin Nutr 62: 712-720.

25. Wolever TM, Spadafora $\mathrm{P}$, Eshuis $\mathrm{H}$ (1991) Interaction between colonic acetate and propionate in humans. Am J Clin Nutr 53: 681-687.

26. Liljeberg HG, Akerberg AK, Bjorck IM (1999) Effect of the glycemic index and content of indigestible carbohydrates of cereal-based breakfast meals on glucose tolerance at lunch in healthy subjects. Am J Clin Nutr 69: 647-655

27. Liljeberg HG, Bjorck IM (1996) Delayed gastric emptying rate as a potentia mechanism for lowered glycemia after eating sourdough bread: studies in humans and rats using test products with added organic acids or an organic salt. Am J Clin Nutr 64: 886-893.

28. Liljeberg HG, Lonner CH, Bjorck IM (1995) Sourdough fermentation or addition of organic acids or corresponding salts to bread improves nutritional properties of starch in healthy humans. J Nutr 125: 1503-1511.

29. Najjar AM, Parsons PM, Duncan AM, Robinson LE, Yada RY, et al. (2009) The acute impact of ingestion of breads of varying composition on blood glucose insulin and incretins following first and second meals. Br J Nutr 101: 391-398. 
Citation: Fletcher JA, Perfield II JW, Thyfault JP, Rector RS (2012) The Second Meal Effect and Its Influence on Glycemia. J Nutr Disorders Ther 2:108. doi:10.4172/2161-0509.1000108

30. Egan JM, Meneilly GS, Habener JF, Elahi D (2002) Glucagon-like peptide-1 augments insulin-mediated glucose uptake in the obese state. J Clin Endocrino Metab 87: 3768-3773.

31. Liljeberg H, Bjorck I (1998) Delayed gastric emptying rate may explain improved glycaemia in healthy subjects to a starchy meal with added vinegar. Eur J Clin Nutr 52: 368-371.

32. Ostman EM, Nilsson M, Liljeberg Elmstahl HGM, Molin G, Bjorck IME (2002) On the effect of lactic acid on blood glucose and insulin responses to cereal products: mechanistic studies in healthy subjects and in vitro. J Cereal Sci 36: 339-346.

33. Ostman EM, Liljeberg Elmstahl HG, Bjorck IM (2002) Barley bread containing lactic acid improves glucose tolerance at a subsequent meal in healthy men and women. J Nutr 132: 1173-1175.

34. Wolever TM, Bentum-Williams A, Jenkins DJ (1995) Physiological modulation of plasma free fatty acid concentrations by diet. Metabolic implications in nondiabetic subjects. Diabetes Care 18: 962-970.

35. Belfort R, Mandarino L, Kashyap S, Wirfel K, Pratipanawatr T, et al. (2005) Dose-response effect of elevated plasma free fatty acid on insulin signaling. Diabetes 54:1640-1648.
36. Tschritter O, Fritsche A, Thamer C, Haap M, Shirkavand F, et al. (2003) Plasma adiponectin concentrations predict insulin sensitivity of both glucose and lipid metabolism. Diabetes 52: 239-243.

37. van Hall G, Steensberg A, Sacchetti M, Fischer C, Keller C, et al. (2003) Interleukin-6 stimulates lipolysis and fat oxidation in humans. J Clin Endocrinol Metab 88: 3005-3010.

38. Edholm T, Degerblad M, Grybäck P, Hilsted L, Holst JJ, et al. (2010) Differential incretin effects of GIP and GLP-1 on gastric emptying, appetite, and insulinglucose homeostasis. Neurogastroenterol Motil 22: 1191-1200.

39. Clark CA Gardiner J, McBurney MI, Anderson S, Weatherspoon LJ, et al. (2006) Effects of breakfast meal composition on second meal metabolic responses in adults with Type 2 diabetes mellitus. Eur J Clin Nutr 60: 1122-1129.

40. Axelsen M, Arvidsson Lenner R, Lonnroth P, Smith U (1999) Breakfast glycaemic response in patients with type 2 diabetes: effects of bedtime dietary carbohydrates. Eur J Clin Nutr 53: 706-710.

41. Jovanovic A, Gerrard J, Taylor R (2009) The second-meal phenomenon in type 2 diabetes. Diabetes Care 32: 1199-1201.

42. Jenkins DJ, Wolever TM, Taylor RH, Griffiths C, Krzeminska K, et al. (1982) Slow release dietary carbohydrate improves second meal tolerance. Am J Clin Nutr 35: 1339-1346. 